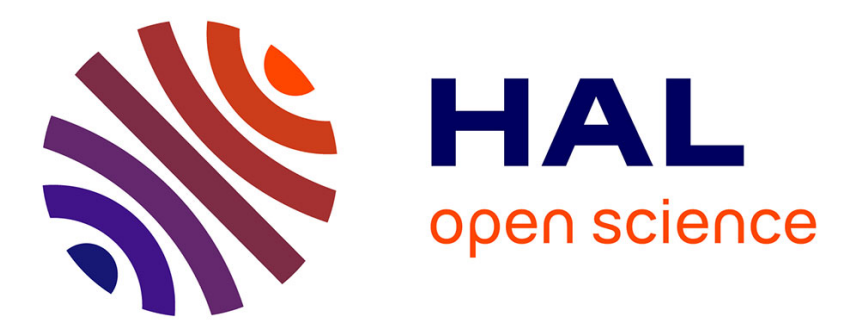

\title{
Marshall lemma in discrete convex estimation
}

Fadoua Balabdaoui, Cécile Durot

\section{To cite this version:}

Fadoua Balabdaoui, Cécile Durot. Marshall lemma in discrete convex estimation. Statistics and Probability Letters, 2015, 10.1016/j.spl.2015.01.016 . hal-01250085

\section{HAL Id: hal-01250085 \\ https://hal.science/hal-01250085}

Submitted on 4 Jan 2016

HAL is a multi-disciplinary open access archive for the deposit and dissemination of scientific research documents, whether they are published or not. The documents may come from teaching and research institutions in France or abroad, or from public or private research centers.
L'archive ouverte pluridisciplinaire HAL, est destinée au dépôt et à la diffusion de documents scientifiques de niveau recherche, publiés ou non, émanant des établissements d'enseignement et de recherche français ou étrangers, des laboratoires publics ou privés. 


\title{
Marshall Lemma in discrete convex estimation
}

\author{
Fadoua Balabdaoui ${ }^{1}$ and Cécile Durot ${ }^{2 *}$
}

January 16, 2015

${ }^{1}$ CEREMADE, Université Paris-Dauphine, 75775, CEDEX 16, Paris, France

${ }^{2}$ UFR SEGMI, Université Paris Ouest Nanterre La Défense, F-92001, Nanterre, France

\begin{abstract}
We show that the supremum distance between the cumulative distribution of the convex LSE and an arbitrary distribution function $F$ with a convex pmf on $\mathbb{N}$ is at most twice the supremum distance between the empirical distribution function and $F$.
\end{abstract}

Keywords: convex, nonparametric least squares, Marshall lemma, pmf, shape constraints

\section{Introduction}

\subsection{A brief overview}

The first Marshall's inequality goes back to Marshall (1970). It states that if $\widehat{F}_{n}$ is the least concave majorant (LCM) of the empirical distribution function $\mathbb{F}_{n}$, then $\left\|\widehat{F}_{n}-F\right\|_{\infty} \leq \| \mathbb{F}_{n}-$

${ }^{*}$ Corresponding author. Email address: cecile.durot@gmail.com 
$F \|_{\infty}$ for an arbitrary concave distribution function $F$. Here, all the distributions involved are supported on $[0, \infty)$. The proof of this inequality is rather elementary and uses basic properties of the LCM. When $F$ is the true distribution function with a decreasing density $f$ supported on a compact real interval and assumed to be continuously differentiable with a strictly negative derivative, Kiefer and Wolfowitz (1976) showed that the global rate of convergence of $\left\|\widehat{F}_{n}-\mathbb{F}_{n}\right\|_{\infty}$ is of order $n^{-2 / 3} \log (n)$ almost surely. In convex density estimation, two Marshall-type of inequalities have been established for the convex least squares estimator (LSE) of a density on $[0, \infty)$ defined by Groeneboom et al. (2001). Let $F$ be an arbitrary distribution function on $[0, \infty)$ such that $F^{\prime}$ is convex, $\mathbb{F}_{n}$ the empirical distribution function, $\widehat{F}_{n}$ the cumulative distribution of the LSE, $\widehat{H}_{n}=\int_{0} \widehat{F}_{n}(s) d s$, $\mathbb{H}_{n}=$ $\int_{0}^{\cdot} \mathbb{F}_{n}(s) d s$ and $H=\int_{0}^{\cdot} F(s) d s$ respectively. Then, Dümbgen et al. (2007) and Balabdaoui and Rufibach (2008) showed that $\left\|\widehat{F}_{n}-F\right\|_{\infty} \leq 2\left\|\mathbb{F}_{n}-F\right\|_{\infty}$, and $\left\|\widehat{H}_{n}-H\right\|_{\infty} \leq\left\|\mathbb{H}_{n}-H\right\|_{\infty}$ respectively. Those results were used by Balabdaoui and Wellner (2007) to show KieferWolfowitz-type of inequalities. Specifically, they show that when $F$ is the true distribution such that $f=F^{\prime}$ is twice continuously differentiable with $f^{\prime \prime}>0,\left\|\widehat{F}_{n}-\mathbb{F}_{n}\right\|_{\infty}$ and $\| \widehat{H}_{n}-$ $\mathbb{H}_{n} \|_{\infty}$ are respectively of order $\left(n^{-1} \log (n)\right)^{3 / 5}$ and $\left(n^{-1} \log (n)\right)^{4 / 5}$.

\subsection{Discrete versus continuous}

In both the monotone and convex estimation problems, the Kiefer-Wolfowitz-type of inequalities recalled above clearly suggest that if one is ready to assume that the true density or its derivative does not admit any flat part on its support, then the empirical and constrained estimators for the unknown distribution function are equivalent for $n$ large enough. In the discrete setting, this is not true anymore simply because the notion of a strict cur-

vature does not exist in this case. In fact, with $\mathbb{F}_{n}$ the empirical distribution and $\widehat{F}_{n}$ the cumulative distribution of the LSE studied by Durot et al. (2013) and Balabdaoui et al. 
(2014), it can be shown with similar arguments as for the proof of Theorem 3.2 in Balabdaoui et al. (2014), that $\left(\widehat{F}_{n}, F_{n}\right)$ converges jointly, at the rate $\sqrt{n}$, to a quite complicated limiting distribution, so that $\sqrt{n}\left(\widehat{F}_{n}-F_{n}\right)$ converges to a distribution that we conjecture is not degenerate. Hence, it is somehow expected that the difference between $\widehat{F}_{n}$ and $\mathbb{F}_{n}$ converges exactly at the rate $1 / \sqrt{n}$ in this case. Although we do not pursue this in this short note, an immediate consequence of our main theorem is that this difference is at most $O_{p}(1 / \sqrt{n})$ in the supremum norm. This theorem gives the same form of the Marshall inequality proved by Dümbgen et al. (2007) for the convex LSE in the continuous setting. Although we re-adapt their idea of using concavity of the difference between the LSE and the density of $F$ (with respect to the counting measure in our case) between two successive knot points $a<b$, our proof does not rely on the equalities $\mathbb{F}_{n}(a)=\widehat{F}_{n}(a)$ and $\mathbb{F}_{n}(b)=\widehat{F}_{n}(b)$ as they are no longer true for the discrete LSE. One has also to deal with discrete sums instead of integrals, which makes the final inequalities a bit less straightforward to obtain. One of the consequences of this note is to be able to assert that the convergence rate of $\widehat{F}_{n}$ to the truth $F$ is the expected rate $1 / \sqrt{n}$, whether $F$ has a finite support or not. In Balabdaoui et al. (2014), the assumption that $F$ is finitely supported on $\{0, \ldots, S\}$ for some unknown integer $S>0$ was made to be able to establish the limiting distribution of the LSE, the general case being much more complex to handle. However, this assumption was not at all necessary for Durot et al. (2013) to obtain that $\left\|\widehat{p}_{n}-p\right\|_{k}=O_{p}(1 / \sqrt{n})$ for all $k \in[2, \infty]$, where $\widehat{p}_{n}$ and $p$ are respectively the convex discrete LSE and the true probability mass function (pmf). However, for $k=\infty$, the transition from this result to showing that $\left\|\widehat{F}_{n}-F\right\|_{\infty}=O_{p}(1 / \sqrt{n})$ is not immediate unless $F$ admits a finite support. The Marshall lemma established in this note alleviates any existing doubt that the parametric convergence rate holds true independently of the nature of the support of the true convex pmf. 


\section{Marshall inequality}

Based on a $n$-sample from a convex discrete probability mass function (pmf) on $\mathbb{N}$, let $\widehat{p}_{n}$ denote the discrete convex LSE of the pmf as defined by Durot et al. (2013). We recall that the points in $\mathbb{N} \backslash\{0\}$ where $\widehat{p}_{n}$ changes its slopes are called knots, and that the characterization of $\widehat{p}_{n}$ is given by

$$
\sum_{x=0}^{z-1} \widehat{F}_{n}(x)\left\{\begin{array}{l}
\geq \sum_{x=0}^{z-1} \mathbb{F}_{n}(x), z \in \mathbb{N} \backslash\{0\} \\
=\sum_{x=0}^{z-1} \mathbb{F}_{n}(x), \text { if } z \text { is a knot of } \widehat{p}_{n},
\end{array}\right.
$$

where $\mathbb{F}_{n}$ is the empirical distribution function and $\widehat{F}_{n}$ is the distribution function corresponding to $\widehat{p}_{n}$, see Proposition 2.1 in Balabdaoui et al. (2014). In the sequel, $F$ is a distribution function on $\mathbb{N}$ with corresponding pmf, $p$, that is decreasing and convex on $\mathbb{N}$. We denote by $\mathcal{K}_{n}$ the set consisting of the point 0 and all knots of $\widehat{p}_{n}$ and by $\bar{p}_{n}$ the empirical pmf, that is the pmf corresponding to $\mathbb{F}_{n}$. We start with the following result.

Lemma 2.1 For $\tau \in \mathcal{K}_{n}$, we have $\widehat{F}_{n}(\tau-1) \leq \mathbb{F}_{n}(\tau-1)$ and $0 \leq \widehat{F}_{n}(\tau)-\mathbb{F}_{n}(\tau) \leq$ $\widehat{p}_{n}(\tau)-\bar{p}_{n}(\tau)$.

Proof. First, suppose that $\tau \geq 2$. From the characterization in (2.1), it follows that

$$
\begin{aligned}
& \sum_{k=0}^{\tau-1} \widehat{F}_{n}(k)=\sum_{k=0}^{\tau-1} \mathbb{F}_{n}(k) \\
& \sum_{k=0}^{\tau-2} \widehat{F}_{n}(k) \geq \sum_{k=0}^{\tau-2} \mathbb{F}_{n}(k)
\end{aligned}
$$

Then, (2.2) - (2.3) yields $\widehat{F}_{n}(\tau-1) \leq \mathbb{F}_{n}(\tau-1)$. Likewise, (2.2) combined to (2.1) with $z=\tau+1$ yields $\mathbb{F}_{n}(\tau) \leq \widehat{F}_{n}(\tau)$. As the inequality $\widehat{F}_{n}(\tau-1) \leq \mathbb{F}_{n}(\tau-1)$ can be rewritten 
under the alternative form $\widehat{F}_{n}(\tau) \leq \mathbb{F}_{n}(\tau)+\widehat{p}_{n}(\tau)-\bar{p}_{n}(\tau)$, this gives the result for $\tau \geq 2$.

If $\tau=1$, then the equality in (2.2) gives the first claimed inequality of the lemma $\widehat{F}_{n}(0)=\mathbb{F}_{n}(0)$. Combined with the inequality in (2.1) with $z=2$, this yields the second claimed inequality of the lemma, that is, $\mathbb{F}_{n}(1) \leq \widehat{F}_{n}(1)=\mathbb{F}_{n}(1)+\widehat{p}_{n}(1)-\bar{p}_{n}(1)$.

Finally, if $\tau=0$, then $\widehat{F}_{n}(-1)=\mathbb{F}_{n}(-1)=0$ and (2.1) with $z=1$ yields $0 \leq \widehat{F}_{n}(0)-$ $\mathbb{F}_{n}(0)=\widehat{p}_{n}(0)-\bar{p}_{n}(0)$ which completes the proof of the Lemma.

In what follows, we will establish two important inequalities linking the extrema of $\widehat{F}_{n}-F$ to those of $\mathbb{F}_{n}-F$.

Proposition 2.2 We have

$$
\max _{x \in \mathbb{N}}\left(\widehat{F}_{n}(x)-F(x)\right) \leq 2 \max _{x \in \mathbb{N}}\left|\mathbb{F}_{n}(x)-F(x)\right|
$$

and

$$
\min _{x \in \mathbb{N}}\left(\widehat{F}_{n}(x)-F(x)\right) \geq-2 \max _{x \in \mathbb{N}}\left|\mathbb{F}_{n}(x)-F(x)\right|
$$

Proof. Put $D=\widehat{F}_{n}-F$ and $d=\widehat{p}_{n}-p$. We begin with the proof of (2.4).

Assume first that the supremum of $D$ over $\mathbb{N}$ is achieved at some $m \geq \widehat{s}_{n}$, where $\widehat{s}_{n}$ is the greatest point in the support of $\widehat{p}_{n}$. Note that $\widehat{s}_{n}+1$ is the largest knot of $\widehat{p}_{n}$. It follows from Theorem 1 of Durot et al. (2013) that $\widehat{s}_{n} \geq \max \left(X_{1}, \ldots, X_{n}\right)$, and $\widehat{F}_{n}(x)=\mathbb{F}_{n}(x)=1$ for all $x \geq \widehat{s}_{n}$, and hence

$$
\begin{aligned}
\sup _{x \in \mathbb{N}}\left(\widehat{F}_{n}(x)-F(x)\right) & =\sup _{x \geq \widehat{s}_{n}}(1-F(x)) \\
& \leq \sup _{x \in \mathbb{N}}\left|\mathbb{F}_{n}(x)-F(x)\right|
\end{aligned}
$$


implying the inequality in (2.4). In the sequel, we assume that the supremum is achieved at some $m<\widehat{s}_{n}$ and we denote by $\tau_{1}$ and $\tau_{2}$ two successive points in $\mathcal{K}_{n}$ such that $\tau_{1} \leq m<\tau_{2}$. If $m=\tau_{2}-1$, then by the first inequality of Lemma 2.1 we can write

$$
\begin{aligned}
\widehat{F}_{n}(m)-F(m) & \leq \mathbb{F}_{n}(m)-F(m) \\
& \leq \sup _{x \in \mathbb{N}}\left|\mathbb{F}_{n}(x)-F(x)\right|
\end{aligned}
$$

implying (2.4). Thus, we assume in the sequel that the maximum of $D$ over $\mathbb{N}$ is achieved at some $m \in\left\{\tau_{1}, \ldots, \tau_{2}-2\right\}$ for some consecutive points $\tau_{1}$ and $\tau_{2}$ in $\mathcal{K}_{n}$ that satisfy $\tau_{2} \geq \tau_{1}+2$. Since the inequality in $(2.4)$ is trivial in the case where $D(m) \leq 0$, we assume furthermore that $D(m)>0$. Since $D(-1)=0$, it follows from the definition of $m$ that $D(m) \geq D(m+1)$ and $D(m) \geq D(m-1)$, which in turn implies that

$$
\widehat{p}_{n}(m) \geq p(m) \text { and } \widehat{p}_{n}(m+1) \leq p(m+1)
$$

Consider now the piecewise linear function $\tilde{d}$ defined on the real interval $\left[\tau_{1}, \tau_{2}\right]$ such that $\tilde{d}=d$ on the set of integers $\left\{\tau_{1}, \ldots, \tau_{2}\right\}$ and which linearly interpolates $\tilde{d}$ between the integers. The inequalities in (2.6) and continuity of $\tilde{d}$ imply that there exists a real number $m_{0} \in[m, m+1]$ such that $\tilde{d}\left(m_{0}\right)=0$. Note that since $p$ is a convex pmf and $\widehat{p}_{n}$ is linear on $\left\{\tau_{1}, \ldots, \tau_{2}\right\}, \tilde{d}$ is concave on $\left[\tau_{1}, \tau_{2}\right]$.

Using the same idea as in Dümbgen et al. (2007), consider the auxiliary linear function $\overline{\tilde{d}}$ defined on the real interval $\left[\tau_{1}, \tau_{2}\right]$ such that

$$
\overline{\tilde{d}}\left(m_{0}\right)=0, \text { and } \sum_{x=m+1}^{\tau_{2}} \overline{\tilde{d}}(x)=D\left(\tau_{2}\right)-D(m)
$$


Thus $\overline{\tilde{d}}(x)=\alpha\left(x-m_{0}\right)$, where the slope $\alpha$ is given by

$$
\alpha=\frac{D\left(\tau_{2}\right)-D(m)}{\sum_{x=m+1}^{\tau_{2}}\left(x-m_{0}\right)}=\frac{2\left(D\left(\tau_{2}\right)-D(m)\right)}{\left(\tau_{2}-m\right)\left(\tau_{2}+m+1-2 m_{0}\right)} .
$$

Since $\tilde{d}-\overline{\tilde{d}}$ is concave on $\left[m_{0}, \tau_{2}\right]$ with a zero at $m_{0}$, it follows that it can only bear a unique sign change on that interval. Therefore, its discretized version $d-\bar{d}$ such that $\bar{d}$ is defined as $\bar{d}(x)=\overline{\tilde{d}}(x)=\alpha\left(x-m_{0}\right)$ on the set of integers $\left\{m+1, \ldots, \tau_{2}\right\}$ can only bear a unique sign change on that set. This in turn implies that

$$
\sum_{x=m+1}^{z}(d(x)-\bar{d}(x)) \geq 0, \text { for all } z \in\left\{m+1, \ldots, \tau_{2}\right\}
$$

To see this, note that there exists $z_{0} \in\left[m_{0}, \tau_{2}\right]$ such that $\tilde{d}-\overline{\tilde{d}} \geq 0$ on $\left[m_{0}, z_{0}\right]$ and $<0$ on $\left(z_{0}, \tau_{2}\right]$. It follows that $d(x)-\bar{d}(x) \geq 0$ if $x \in\left\{m+1, \ldots,\left\lfloor z_{0}\right\rfloor\right\}$ and $d(x)-\bar{d}(x)<0$ if $x \in\left\{\left\lfloor z_{0}\right\rfloor+1, \ldots, \tau_{2}\right\}$, where $\lfloor t\rfloor$ denotes the integer part of a real number $t$. Hence, the inequality in (2.8) holds true for $z \in\left\{m+1, \ldots,\left\lfloor z_{0}\right\rfloor\right\}$. Now, the second equality in $(2.7)$ implies that if $z \in\left\{\left\lfloor z_{0}\right\rfloor+1, \ldots, \tau_{2}-1\right\}$, then

$$
\sum_{x=m+1}^{z}(d(x)-\bar{d}(x))=-\sum_{x=z+1}^{\tau_{2}}(d(x)-\bar{d}(x))>0
$$

and if $z=\tau_{2}$, then $\sum_{x=m+1}^{z}(d(x)-\bar{d}(x))=0$. Thus, we can write for all $z \in\left\{m+1, \ldots, \tau_{2}\right\}$

$$
D(z)-D(m) \geq \sum_{x=m+1}^{z} \bar{d}(x)=\alpha \sum_{x=m+1}^{z}\left(x-m_{0}\right)
$$

or equivalently

$$
D(z) \geq D(m)+\left(D\left(\tau_{2}\right)-D(m)\right) \frac{(z-m)\left(z+m+1-2 m_{0}\right)}{\left(\tau_{2}-m\right)\left(\tau_{2}+m+1-2 m_{0}\right)} .
$$


On the other hand, taking the difference between the equality and inequality of the characterization in (2.1) at the points $z=\tau_{2}$ and $z=m+1$ respectively we can write

$$
\sum_{x=m+1}^{\tau_{2}-1} D(x) \leq \sum_{x=m+1}^{\tau_{2}-1}\left(\mathbb{F}_{n}(x)-F(x)\right)
$$

which, combined with (2.9), implies that

$$
\sum_{x=m+1}^{\tau_{2}-1}\left(\mathbb{F}_{n}(x)-F(x)\right) \geq\left(\tau_{2}-1-m\right) D(m)+\left(D\left(\tau_{2}\right)-D(m)\right) S
$$

where

$$
\begin{aligned}
S & =\frac{\sum_{z=m+1}^{\tau_{2}-1}(z-m)(z-m+1)-2\left(m_{0}-m\right) \sum_{z=m+1}^{\tau_{2}-1}(z-m)}{\left(\tau_{2}-m\right)\left(\tau_{2}+m+1-2 m_{0}\right)} \\
& =\frac{\sum_{k=1}^{\tau_{2}-1-m} k^{2}+\sum_{k=1}^{\tau_{2}-1-m} k-2\left(m_{0}-m\right) \sum_{k=1}^{\tau_{2}-1-m} k}{\left(\tau_{2}-m\right)\left(\tau_{2}+m+1-2 m_{0}\right)} \\
& =\frac{\frac{1}{3}\left(\tau_{2}-1-m\right)\left(\tau_{2}-m\right)\left(\tau_{2}-m-\frac{1}{2}\right)+\left(\tau_{2}-1-m\right)\left(\tau_{2}-m\right)\left(\frac{1}{2}-\left(m_{0}-m\right)\right)}{\left(\tau_{2}-m\right)\left(\tau_{2}+m+1-2 m_{0}\right)} \\
& =\frac{1}{3}\left(\tau_{2}-1-m\right) \times \frac{\tau_{2}-m+1-3 t}{\tau_{2}-m+1-2 t}, \quad \text { where } t=m_{0}-m \in[0,1] .
\end{aligned}
$$

Since $D\left(\tau_{2}\right)-D(m) \leq 0$ by definition of $m$, we want to find an upper bound for $S$ in $t \in[0,1]$. To make the notation even lighter, let us put $\eta=\tau_{2}-m+1$. Note that $m \leq \tau_{2}-2$ by assumption so that $\eta>2$ and $\eta-2 t>0$ for all $t \in[0,1]$. A quick study of the variations of the function $t \mapsto(\eta-3 t) /(\eta-2 t)$, for $t \in[0,1]$ shows that it attains its maximal value at 0 and that the maximal value is equal to 1 . Hence, $S \leq\left(\tau_{2}-1-m\right) / 3$ so that the inequality in (2.10) implies that

$$
\sum_{x=m+1}^{\tau_{2}-1}\left(\mathbb{F}_{n}(x)-F(x)\right) \geq\left(\tau_{2}-1-m\right) D(m)+\frac{D\left(\tau_{2}\right)-D(m)}{3}\left(\tau_{2}-1-m\right) .
$$


Now, using the fact that

$$
\sum_{x=m+1}^{\tau_{2}-1}\left(\mathbb{F}_{n}(x)-F(x)\right) \leq\left(\tau_{2}-1-m\right) \max _{x \in \mathbb{N}}\left(\mathbb{F}_{n}(x)-F(x)\right)
$$

it follows that

$$
D(m) \leq \frac{3}{2} \max _{x \in \mathbb{N}}\left(\mathbb{F}_{n}(x)-F(x)\right)-\frac{1}{2} D\left(\tau_{2}\right)
$$

Combining the second statement of Lemma 2.1 with (2.11) yields

$$
\begin{aligned}
D(m) & \leq \frac{3}{2} \max _{x \in \mathbb{N}}\left(\mathbb{F}_{n}(x)-F(x)\right)+\frac{1}{2} \max _{x \in \mathbb{N}}\left|\mathbb{F}_{n}(x)-F(x)\right| \\
& \leq 2 \max _{x \in \mathbb{N}}\left|\mathbb{F}_{n}(x)-F(x)\right|
\end{aligned}
$$

which completes the proof of (2.4).

Now we turn to the proof of (2.5). Similar to the proof of (2.4), it is easy to see that (2.5) holds if the minimum of $D$ is achieved at some $m \geq \widehat{s}_{n}$. Therefore, it suffices to prove (2.5) for the case where the minimum of $D$ over $\mathbb{N}$ is achieved at some $m<\widehat{s}_{n}$. As done above, we denote by $\tau_{1}$ and $\tau_{2}$ two successive points in $\mathcal{K}_{n}$ such that $\tau_{1} \leq m<\tau_{2}$. If $m=\tau_{1}$, then it follows from the second statement of Lemma 2.1 that

$$
\begin{aligned}
\widehat{F}_{n}(m)-F(m) & \geq \mathbb{F}_{n}(m)-F(m) \\
& \geq-\max _{x \in \mathbb{N}}\left|\mathbb{F}_{n}(x)-F(x)\right|
\end{aligned}
$$

implying (2.5). Thus, we assume in the sequel that the minimum of $D$ over $\mathbb{N}$ is achieved at some $m \in\left\{\tau_{1}+1, \ldots, \tau_{2}\right\}$ for some consecutive points $\tau_{1}$ and $\tau_{2}$ in $\mathcal{K}_{n}$ that satisfy $\tau_{1} \leq \tau_{2}-1$. By definition of $m$, we have $D(m) \leq D(m+1)$ and $D(m) \leq D(m-1)$, which 
imply that

$$
\widehat{p}_{n}(m) \leq p(m) \text { and } \widehat{p}_{n}(m+1) \geq p(m+1)
$$

Consider now the piecewise linear function $\tilde{d}$ defined on $\left[\tau_{1}, \tau_{2}\right]$ such that $\tilde{d}=d$ on the set of integers $\left\{\tau_{1}, \ldots, \tau_{2}\right\}$ and which linearly interpolates $d$ between the integers. The inequalities in (2.13) and continuity of $\tilde{d}$ imply that there exists a real number $m_{0} \in[m, m+1]$ such that $\tilde{d}\left(m_{0}\right)=0$. As in the proof of $(2.4)$, consider again the auxiliary linear function $\overline{\tilde{d}}$ defined on $\left[\tau_{1}, \tau_{2}\right]$ such that

$$
\overline{\tilde{d}}\left(m_{0}\right)=0, \text { and } \sum_{x=\tau_{1}}^{m} \overline{\tilde{d}}(x)=D(m)-D\left(\tau_{1}-1\right)
$$

Thus $\overline{\tilde{d}}(x)=\alpha\left(x-m_{0}\right)$, where

$$
\alpha=\frac{2\left(D(m)-D\left(\tau_{1}-1\right)\right)}{\left(m-\tau_{1}+1\right)\left(\tau_{1}+m-2 m_{0}\right)} .
$$

Using the fact that $\tilde{d}-\overline{\tilde{d}}$ is concave on the real interval $\left[\tau_{1}, \tau_{2}\right]$ with a zero at $m_{0} \in[m, m+1]$, it follows that its discretized version $d-\bar{d}$ can only bear a unique sign change on the set of integers $\left\{\tau_{1}, \ldots, m\right\}$. This in turn implies that we have

$$
\sum_{x=z}^{m}(d(x)-\bar{d}(x)) \geq 0, \text { for all } z \in\left\{\tau_{1}, \ldots, m\right\}
$$

Thus, we can write for all $z \in\left\{\tau_{1}, \ldots, m\right\}$

$$
D(m)-D(z-1) \geq\left(D(m)-D\left(\tau_{1}-1\right)\right) \frac{(m-z+1)\left(m+z-2 m_{0}\right)}{\left(m-\tau_{1}+1\right)\left(m+\tau_{1}-2 m_{0}\right)}
$$


or equivalently

$$
D(y) \leq D(m)-\left(D(m)-D\left(\tau_{1}-1\right)\right) \frac{(m-y)\left(m+y+1-2 m_{0}\right)}{\left(m-\tau_{1}+1\right)\left(m+\tau_{1}-2 m_{0}\right)}
$$

for $y \in\left\{\tau_{1}-1, \ldots, m-1\right\}$. On the other hand, taking the difference between the equality and inequality of the characterization in (2.1) at the points $z=\tau_{1}$ and $z=m$ respectively implies that

$$
\sum_{x=\tau_{1}}^{m-1} D(x) \geq \sum_{x=\tau_{1}}^{m-1}\left(\mathbb{F}_{n}(x)-F(x)\right)
$$

which combined with (2.14) yields

$$
\sum_{x=\tau_{1}}^{m-1}\left(\mathbb{F}_{n}(x)-F(x)\right) \leq\left(m-\tau_{1}\right) D(m)-\left(D(m)-D\left(\tau_{1}-1\right)\right) S
$$

where

$$
S=\frac{1}{3}\left(m-\tau_{1}\right) \frac{\eta+3 t}{\eta+1+2 t} \leq \frac{1}{3}\left(m-\tau_{1}\right)
$$

with $\eta=m-\tau_{1}-1$ and $t=m_{0}-m \in[0,1]$. Now, using the fact that

$$
\sum_{x=\tau_{1}}^{m-1}\left(\mathbb{F}_{n}(x)-F(x)\right) \geq\left(m-\tau_{1}\right) \min _{x \in \mathbb{N}}\left(\mathbb{F}_{n}(x)-F(x)\right)
$$

it follows that

$$
\begin{aligned}
\min _{x \in \mathbb{N}}\left(\mathbb{F}_{n}(x)-F(x)\right) & \leq D(m)-\frac{1}{3}\left(D(m)-D\left(\tau_{1}-1\right)\right)=\frac{2}{3} D(m)+\frac{1}{3} D\left(\tau_{1}-1\right) \\
& \leq \frac{2}{3} D(m)+\frac{1}{3}\left(\mathbb{F}_{n}\left(\tau_{1}-1\right)-F\left(\tau_{1}-1\right)\right)
\end{aligned}
$$


where the last inequality is an immediate consequence of the first equality of Lemma 2.1. Hence,

$$
D(m) \geq \frac{3}{2} \min _{x \in \mathbb{N}}\left(\mathbb{F}_{n}(x)-F(x)\right)-\frac{1}{2}\left(\mathbb{F}_{n}\left(\tau_{1}-1\right)-F\left(\tau_{1}-1\right)\right)
$$

and (2.5) follows.

In what follows, we use $\|q\|_{\infty}$ to denote $\sup _{x \in \mathbb{N}}|q(x)|$ for a function $q$ defined on $\mathbb{N}$. We now state the Marshall lemma for the convex discrete estimator.

Theorem 2.3 For any distribution function $F$ defined on $\mathbb{N}$ whose probability mass function is convex on $\mathbb{N}$, we have $\left\|\widehat{F}_{n}-F\right\|_{\infty} \leq 2\left\|\mathbb{F}_{n}-F\right\|_{\infty}$.

Proof. The claimed inequality is an immediate consequence of Proposition 2.2 and the fact that $\|q\|_{\infty}=\max _{x \in \mathbb{N}} q(x) \vee \max _{x \in \mathbb{N}}(-q(x))=\max _{x \in \mathbb{N}} q(x) \vee\left(-\min _{x \in \mathbb{N}} q(x)\right)$.

\section{References}

Balabdaoui, F., Durot, C. and Koladjo, F. (2014). On asymptotics of the discrete convex lse of a pmf. arXiv preprint arXiv:1404.3094.

Balabdaoui, F. and Rufibach, K. (2008). A second Marshall inequality in convex estimation. Statist. Probab. Lett. 78 118-126.

URL http://dx.doi.org/10.1016/j.spl.2007.05.009

Balabdaoui, F. and Wellner, J. A. (2007). A Kiefer-Wolfowitz theorem for convex densities. In Asymptotics: particles, processes and inverse problems, vol. 55 of IMS Lecture Notes Monogr. Ser. Inst. Math. Statist., Beachwood, OH, 1-31.

URL http://dx.doi.org/10.1214/074921707000000256 
Dümbgen, L., Rufibach, K. and Wellner, J. A. (2007). Marshalls lemma for convex density estimation. In Asymptotics: Particles, Processes and Inverse Problems. Institute of Mathematical Statistics, 101-107.

Durot, C., Huet, S., Koladjo, F. and Robin, S. (2013). Least-squares estimation of a convex discrete distribution. Comput. Statist. Data Anal. 67 282-298. URL http://dx.doi.org/10.1016/j.csda.2013.04.019

Groeneboom, P., Jongbloed, G. and Wellner, J. A. (2001). Estimation of a convex function: characterizations and asymptotic theory. Ann. Statist. 29 1653-1698.

Kiefer, J. and Wolfowitz, J. (1976). Asymptotically minimax estimation of concave and convex distribution functions. Z. Wahrscheinlichkeitstheorie und Verw. Gebiete 34 $73-85$.

Marshall, A. W. (1970). Discussion of Barlow and van Zwet's paper. In Nonparametric techniques in Statistical Inference, vol. 1969 of Proceedings of the First International Symposium on Nonparametric Techniques held at Indiana University, June. Cambridge University Press, London-New York, 174-176. 